\title{
Preface
}

\section{Detection, Prevention, and Pre-Clinical Treatment of Alzheimer's Disease}

\author{
Jack C. de la Torre* \\ Department of Psychology, University of Texas at Austin, Austin, TX, USA
}

Despite thousands of medical and scientific articles published every year about Alzheimer dementia, it remains a clinically neglected disease. There is no Heisenbergian uncertainty that Alzheimer's disease (AD) has become one of the most ominous medical challenges of the 21 st century. It follows that if rapid action is not taken to substantially reduce its exponential growth, a socio-economic crisis will develop and devour many government-sponsored health plans within the next few decades. So, what do we do about it and how do we do it?

It makes a lot of sense to sort out what has been done in the field of $\mathrm{AD}$ so far and how we might learn from prior failures. The time, effort, and billions of dollars spent by industry in the last twenty years searching for a pharmaceutical nostrum to provide questionable palliation to patients who may not be aware of their surroundings has not helped in the battle to limit the number of people affected by AD. These nostrums, which are charitably called 'cognitive enhancers', do no such thing, not even in treating mild cognitive impairment or slowing-down conversion to $\mathrm{AD}$ [1]. The ethical imperative of this tactic is nothing less than a cruel approach to Alzheimer victims and their care-takers. It also takes advantage of the fact that a dementia remedy does not exist, thereby allowing the void created as an opportunity to try and launch products into the consumer market that are on

\footnotetext{
*Correspondence to: Jack C. de la Torre, MD, PhD, Professor of Psychology (Adjunct), Department of Psychology, University of Texas at Austin, 1 University Station, Austin, TX 78712-0187, USA. Tel.: +1 512475 7596; E-mail: jcdelatorre@ comcast.net.
}

the threshold of quack medicine. According to a recent review of trial drug development in the U.S. between 2002-2012, the Research and Development arena has racked up a colossal $99.6 \%$ clinical trial failure rate [2].

To provide an alternative strategy to this mind-set, a panel of international experts was invited to review, in this Supplemental Issue of the Journal of Alzheimer's Disease (JAD), measures that could help bend the impending impact $\mathrm{AD}$ will have on the rising aging population. The reviews offer useful understanding of the many clinical facets that must be identified, integrated into a plan of action and clinically applied to stop, delay, or reduce AD onset.

Since there is now wide acceptance that AD has a prolonged preclinical phase, three main topics arose from the invited reviews to address this prodromal stage. The topics deal with the detection, prevention, and pre-clinical treatment of conditions that increase the liability of progressive cognitive impairment prior to AD onset. The first topic, detection, deals with tools that test and measure the early indicators which signal cognitive decline. The second topic considers primary and secondary prevention of AD as the desired goal of controlling this disorder. Primary prevention should ideally focus on blocking the development of known risk factors that can promote dementia.

When primary prevention is not feasible, early secondary prevention of AD should aim at managing the offending risk factors that are detected, prior to cognitive impairment and AD onset. These risk factors include cardiovascular and cerebrovascular disorders 
as well as a variety of brain insults, unhealthy lifestyle, and diet. Detection and prevention of these risk factors are aptly examined and discussed by the panel of experts.

The third topic explores the pre-clinical treatment of $\mathrm{AD}$. This is a growing research field that differs from the present prescriptive drugs available on the U.S. market to modestly manage some AD symptoms. Pre-clinical treatment of AD targets prevention by searching for novel interventions based on the cellular and molecular changes observed in animal models and Alzheimer pathology. There are no interventions presently available to prevent $\mathrm{AD}$ onset. However, these potential treatments could one day become the vaccines of $\mathrm{AD}$.

In the U.S., one major problem in advancing our understanding of $\mathrm{AD}$ and exploiting the knowledge gained, is a poverty of research funding by the National Institutes of Health, whose investment in Alzheimer research was $\$ 484$ million for fiscal year 2013. Instead of getting the best bang for its bucks by spreading this paltry sum among good applicant researchers seeking new ideas, a half dozen favorite investigators were identified by an undisclosed process and awarded over $\$ 65$ million of the AD budget over a 5-year period [3].

The feeble Alzheimer funding has also plagued private foundations. For example, the non-profit, publicly-funded Alzheimer's Association in Chicago now has several advisors who 'preselect' which researchers will be 'allowed' to submit a grant proposal for possible funding. The danger behind this approach is to rely on the wisdom of advisors who cherry-pick which AD investigations fit their box. This shallow reasoning essentially squelches the puzzle-solving activity that is the basis of scientific discovery and which often does not fit a box but nonetheless has led to major medical breakthroughs in the past. A prime example of this error in judgment is the pharmaceutical industry's vested interest in finding a pill that provides 'some' help to Alzheimer victims. It seems irrelevant to their thinking that most Alzheimer patients targeted for such therapy already show irreversible brain degeneration. On the other hand, if market profit is the prime consideration, this may not be seen as an error in judgment but a calculated choice between principle and gain.

These are sad times for people with $\mathrm{AD}$ who have lost their sense of awareness and even sadder for those million plus individuals destined to enter this terrible fold every year. It is also a difficult time for unfunded researchers who cannot join the fight against AD.

It seems an auspicious moment to open a dialogue between those pursuing a treatment for AD and those favoring prevention of this dementia. Such a dialogue could lead to a more effective course of action in confronting the needs of $\mathrm{AD}$ patients and those at risk of developing this disorder. The reviews contained in this supplementary issue of JAD may set the stage for such a discourse and in addition, provide some viable tracks on the road to discovering a realistic pathway for coping with this grim disorder.

\section{REFERENCES}

[1] Tricco AC, Soobiah C, Berliner S, Ho JM, Ng CH, Ashoor HM, Chen MH, Hemmelgarn B, Straus SE (2013) Efficacy and safety of cognitive enhancers for patients with mild cognitive impairment: A systematic review and meta-analysis. CMAJ 185, 1393-1401.

[2] Cummings JL, Morstorf T, Zhong K (2014) Alzheimer's disease drug development pipeline: Few candidates, frequent failures. Alzheimers Res Ther 6, 37.

[3] National Institutes of Health (2013) NIH funding boosts new Alzheimer's research on prevention, novel drug targets. NIH News \& Events, 2013. 